\title{
Distinguishing different dynamics in electroencephalographic time series through a complex network approach
}

\author{
Andriana S. L. O. Campanharo ${ }^{1}$ \\ Departamento de Bioestatística, Instituto de Biociências, UNESP, Botucatu, SP \\ Fernando M. Ramos ${ }^{2}$ \\ Laboratório Associado de Computação e Matemática Aplicada, Instituto Nacional de Pesquisas \\ Espaciais, INPE, São José dos Campos, SP
}

\begin{abstract}
In the context of dynamical systems, time series analysis is frequently used to identify the underlying nature of a phenomenon of interest from a sequence of observations and to forecast future outcomes. Recently, a map from time series to network has been proposed [4], allowing the use of network statistics to characterize time series. In this approach, time series quantiles are naturally mapped into nodes of a graph (here called Quantile Graph - QG). Therefore, the proposed map (here called QG algorithm) is able to capture and quantify features such as long range correlations or randomness present in the underlying dynamics of the original signal, making it a powerful tool for the analysis of nonlinear systems $[4,5]$. As an illustration we applied the QG algorithm to the long standing problem of detecting the differences between electroencephalographic time series (EEG) of healthy and unhealthy subjects. The main goal is to find out how the differences in dynamics are reflected in the corresponding networks. Our results show that resulting networks display clear differences in topology, which are especially apparent on the smallworld behaviour in the network associated with the healthy subject.
\end{abstract}

\section{Introduction}

Epilepsy is a neurological disorder characterized by the presence of recurring seizures. Like many other neurological disorders, epilepsy can be assessed by the electroencephalogram technic. Visual inspection of the EEG data has not yet led to detection of all characteristic changes preceding seizure onsets and detection of seizures by visual inspection of the EEG even by a trained neurologist is challenging for a variety of reasons such

\footnotetext{
${ }^{1}$ andriana@ibb.unesp.br

${ }^{2}$ fernando.ramos@inpe.br
} 
as excessive presence of myogenic artifacts. Hence, several studies have focused on the detection of epilepsy from EEG signals using automatic analysis like methods that detect and quantify non-linear mechanisms and thereby better reflect the characteristics of the EEG signals [3]. Those methods are basically based on surrogate analysis [3] and phasespace technics $[2,7]$. So there is considerable research toward developing novel methods to quantify or capture additional information in EEG time series in new ways [4].

In the last two decades, research on complex networks became the focus of widespread attention, with developments and applications spanning different scientific areas, from sociology and biology to physics [1]. One of the reasons behind the growing popularity of complex networks is that almost any discrete structure can be suitably represented as a graph, whose features may be then characterized, analyzed and, eventually, related to its respective dynamics [6]. Recently an approach has been proposed for mapping a time series into a complex network representation, based on the concept of transition probabilities [4]. This study has shown that distinct features of a time series can be mapped into networks with distinct topological properties, opening the door to the analysis of discrete, timeordered data sets with mathematical tools usually used in the study of geometric shapes and topological spaces. Here we show that the complex network theory can be efficiently used in the problem of detecting differences in EEG signals of patients in different health conditions.

This paper is organized as follows. After this Introduction, we describe in Section 2 the QG method for mapping a time series into a network. Results are presented and discussed in Section 3 while an overall conclusion is given in Section 4.

\section{Methods}

Let the range of values in a time series be coarse-grained into $Q$ quantiles $q_{1}, \ldots, q_{Q}$, and let $\mathcal{M}$ be a map from a time series $X \in \mathcal{T}$ to a network $g \in \mathcal{G}$, with $X=\{x(t) \mid t \in$ $\mathbb{N}, x(t) \in \mathbb{R}\}$ and $g=\{\mathcal{N}, \mathcal{A}\}$ being a set of nodes $\mathcal{N}$ and $\operatorname{arcs} \mathcal{A}$. Specifically, $\mathcal{M}$ assigns each quantile $q_{i}$ to a node $n_{i} \in \mathcal{N}$ in the corresponding network. Two nodes $n_{i}$ and $n_{j}$ are connected with a weighted $\operatorname{arc}\left(n_{i}, n_{j}, w_{i j}\right) \in \mathcal{A}$ whenever two values $x(t)$ and $x(t+1)$ belong respectively to quantiles $q_{i}$ and $q_{j}$, with $t=1,2, \ldots, T$. An illustration of the QG method can be found in Figure 1.

Weights $w_{i j}$ are simply given by the number of times a value in quantile $q_{i}$ at time $t$ is followed by a point in quantile $q_{j}$ at time $t+1$, normalized by the total number of transitions. Repeated transitions through the same arc increase the value of the corresponding weight. With proper normalization, the weighted adjacency matrix becomes a Markov transition matrix $\mathbf{W}_{k}$, with $\sum_{j}^{Q} w_{i j}$. The resulting network is weighted, directed and connected, with $Q$ being typically much smaller than $T$ [4].

Previous works have shown that time series with different properties are mapped into complex networks with different topologies. For example, it was found an association between periodic time series and regular networks, random time series and random networks, pseudo-periodic time series and small-world networks, Brownian motion and chain-like networks $[4,5]$. 


\section{Quantiled values}

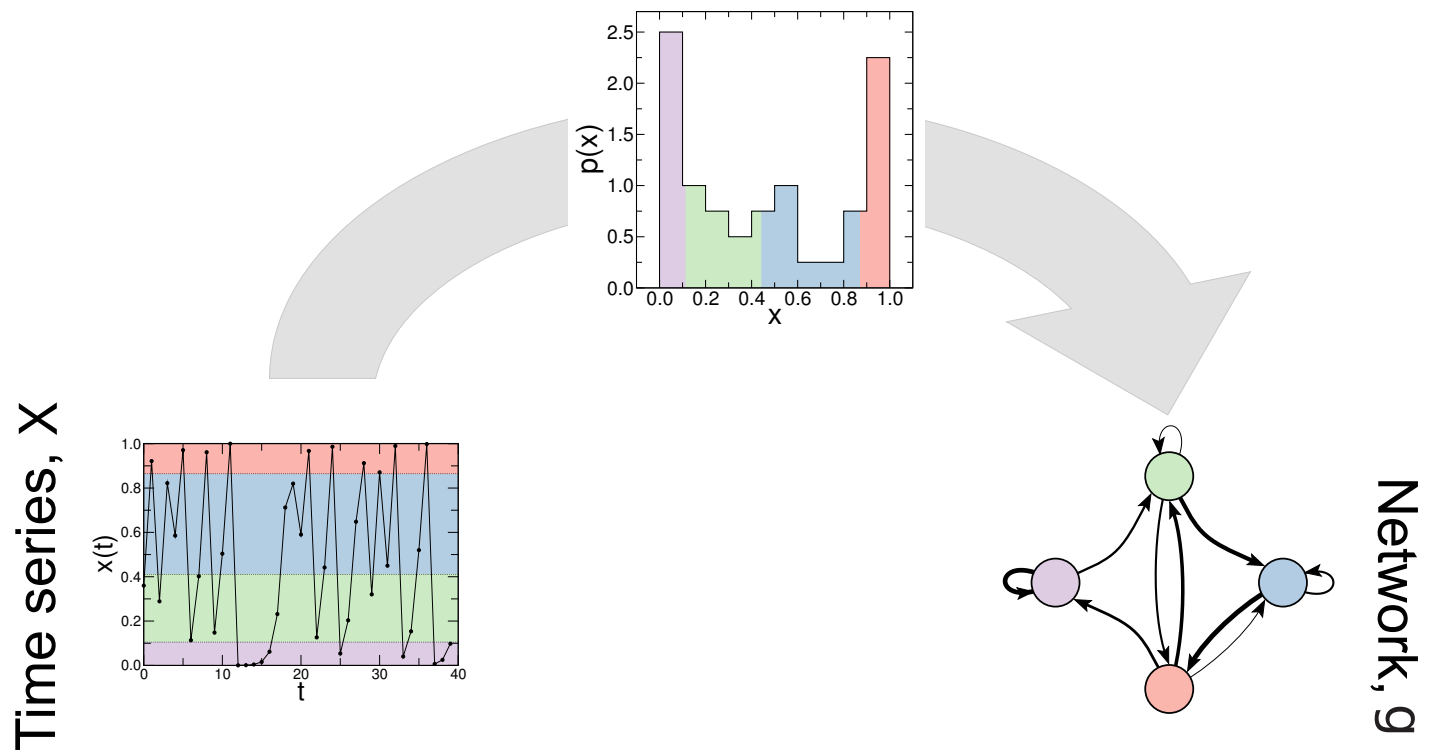

Figure 1: Illustration of the QG method. A time series $X$ with $T=40$ is split into $Q=4$ quantiles (colored shading) and each quantile $q_{i}$ is assigned to a node $n_{i} \in \mathcal{N}$ in the corresponding network $g$. Two nodes $n_{i}$ and $n_{j}$ are then connected in the network with a weighted arc $\left(n_{i}, n_{j}, w_{i j}\right) \in \mathcal{A}$ where the weight $w_{i j}$ of the arc is given by the probability that a point in quantile $q_{i}$ is followed by a point in quantile $q_{j}$. Repeated transitions between quantiles results in arcs in the network with larger weights (represented by thicker lines) and therefore higher values in the corresponding transition matrix [4]. The resulting network has 4 nodes, is weighted, directed and connected.

\section{Results}

We apply the QG algorithm to the long standing problem of detecting the subtle differences between EEG time series of healthy and unhealthy subjects. The EEG data used in this study was taken from the artifact free EEG time series obtained by the Department of Epileptology, in the University of Bonn [3]. Specifically, we randomly selected two EEG time series; one from a healthy subject and the other one from a epilepsy subject within the epileptogenic zone. The EEG signals were recorded at a sampling rate of $173.61 \mathrm{~Hz}$ and consist of 4.097 time points each. Usually, epileptic seizures are caused by disturbed brain activity - which simply means that the normal activity of the brain is suddenly interrupted and changes. The epileptic behavior is then characterized by the presence of several aperiodic spikes in the EEG from the unhealthy subject in Figure 2.

We applied the QG in the healthy and unhealthy EEG time series using 40 quantiles (Fig. 3). The resulting networks display clear differences in topology. The QG related to the healthy subject presents a bulky structure due to the irregular pattern found in the corresponding healthy EEG time series. On the other hand, the QG related to the 
unhealhty subject presents an elongated chain-like pattern due to the bursts present in the corresponding time series.
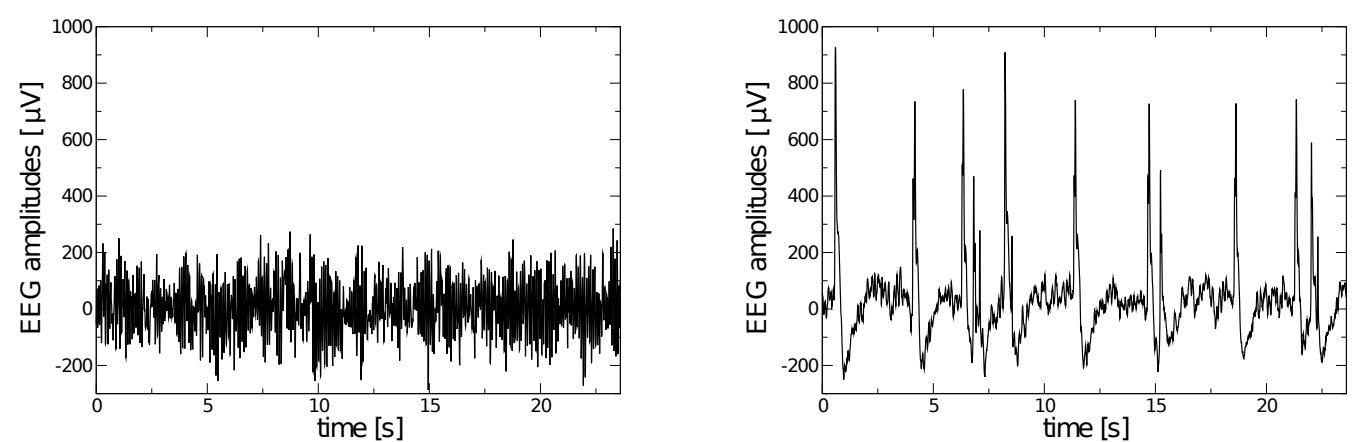

Figure 2: EEG time series with $T=4.097$ from healthy and unhealty subjects, respectively.
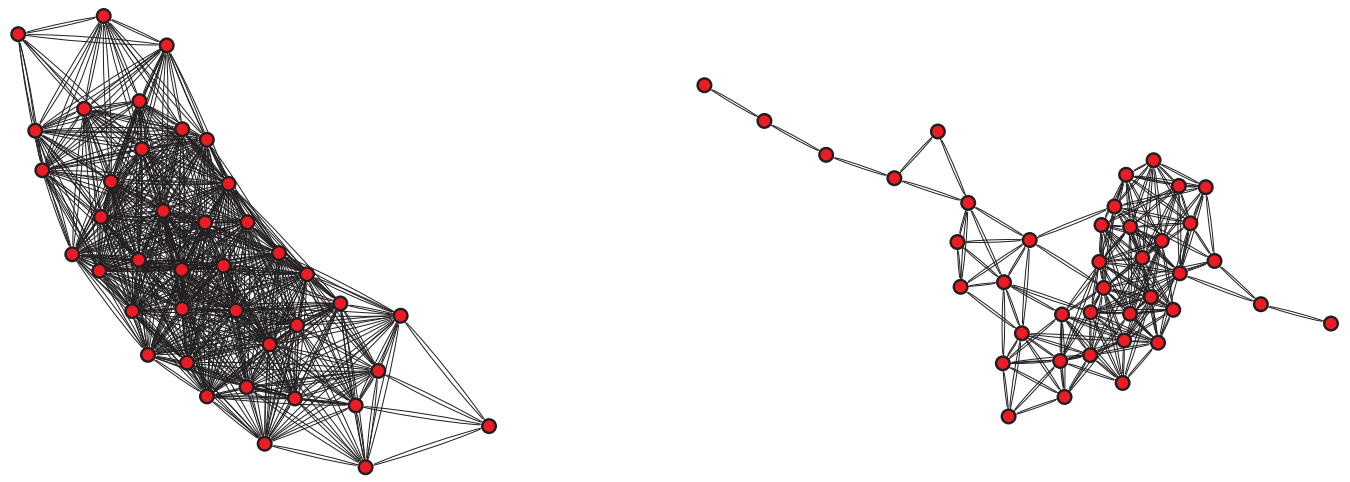

Figure 3: QGs with $Q=40$ obtained from healthy and unhealthy EEG time series, respectively.

In unweighted networks the Clustering Coefficient $(C)$ is a real number between zero and one that is zero when there is no clustering, and one for maximal clustering, which happens when the network consists of disjoint cliques. The Average Path Length $(L)$ is a measure of the efficiency of information on a network and it is defined as the average number of steps along the shortest paths for all possible pairs of network nodes [6]. We quantify the topology differences in the networks showed in Figure 3 by computing their corresponding $C$ and $L$. We also generate the randomized versions of those networks and compute their corresponding Clustering Coefficient $\left(C_{\text {random }}\right)$ and Smallest Path Length $\left(L_{\text {random }}\right)$ (Table 1$)$.

The "healthy" and "unhealthy networks" present different values of $L$ and $C$. More specifically, the value of $L$ is much higher in the "unhealthy network" than in the "healthy 
network" since the chain-like pattern increases the distance between any pair of nodes in the corresponding network. Moreover, the irregular behavior in the healthy network decreases the value of $C$. Comparing $L$ and $C$ with their corresponding $L_{\text {random }}$ and $C_{\text {random }}$ we find out that the two networks belong to different well-known classes in the network theory - the healthy network behaves like a small-world network [8] and unhealthy one like a regular network.

\begin{tabular}{|c|c|c|c|c|}
\hline & $L$ & $L_{\text {random }}$ & $C$ & $C_{\text {random }}$ \\
\hline healthy subject & 3.1474 & 3.0288 & 0.4421 & 0.3739 \\
\hline unhealthy subject & 12.2045 & 8.3731 & 0.5152 & 0.3031 \\
\hline
\end{tabular}

\section{Conclusions}

We have shown that EEG time series of healthy and unhealthy subjects are mapped through the QG method into networks whose topological characteristics are very different. These results attest that the QG method is a useful tool for the analysis of nonlinear dynamics and able to quantify features such as long-range correlations or randomness in complex signal. Our analysis can be easily extended to detect differences in the data structures of many real systems as interbeat interval time series of healthy and unhealthy subjects.

\section{Acknowledgements}

A. S. L. O. C. acknowledges the support of FAPESP: 2014/05145-0 and 2013/19905-3. The support of Conselho Nacional de Desenvolvimento Científico e Tecnológico (CNPq) (Brazil) is acknowledged by F. M. Ramos.

The figures were generated with PyGrace (http://pygrace.github.io/) and Pajek (http://vlado.fmf.uni-lj.si/pub/networks/pajek/).

\section{References}

[1] R. Albert and A. L. Barabási. Statistical Mechanics of Complex Networks, Reviews of Modern Physics, 2002. DOI: 10.1103/RevModPhys.74.47.

[2] R. G. Andrzejak, K. Lehnertz, F. Mormann, C. Rieke, P. David and C. E. Elger. Indications of nonlinear dynamics and finite-dimensional structures in time series of brain electrical activity: Dependence on recording region and brain state, Physical Review E, 2001. DOI: 10.1103/PhysRevE.64.061907. 
[3] R. G. Andrzejak, K. Schindler and C. Rummel. Nonrandomness, nonlinear dependence, and nonstationarity of electroencephalographic recordings from epilepsy patients, Physical Review E, 2012. DOI: 10.1103/PhysRevE.86.046206.

[4] A. S. L. O. Campanharo, M. I. Sirer, R. D. Malmgren, F. M. Ramos and L. A. N. Amaral, Duality between time series and complex networks, PLoS ONE, 2011. DOI: 10.1371/journal.pone.0023378.

[5] A. S. L. O. Campanharo and F. M. Ramos, Hurst exponent estimation of self-affine time series using quantile graphs, Physica A, 2016. DOI: 10.1016/j.physa.2015.09.094.

[6] L. F. Costa, F. A. Rodrigues, G. Travieso and P. R. Villas. Characterization of complex networks: a survey of measurements, Advances in Physics, 2007. DOI: 10.1080/00018730601170527.

[7] I. Vlachos, I. and D. Kugiumtzis. Nonuniform state-space reconstruction and coupling detection, Physical Review E, 2001. DOI: 10.1103/PhysRevE.82.016207.

[8] D. J. Watts and S. H. Strogatz. Collective Dynamics of "Small World" Networks, Letters to Nature, 1998. DOI: 10.1038/30918. 